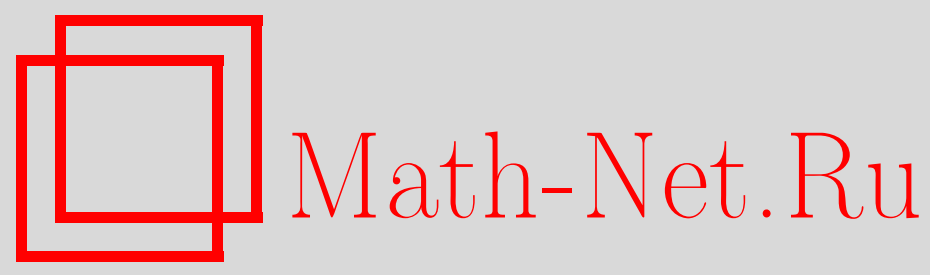

В. Е. Тараканов, О максимальных паросочетаниях в $n$ мерном кубе, Матем. заметки, 2001, том 69, выпуск 3, 454465

DOI: https://doi.org/10.4213/mzm517

Использование Общероссийского математического портала Math-Net.Ru подразумевает, что вы прочитали и согласны с пользовательским соглашением http://www. mathnet.ru/rus/agreement

Параметры загрузки:

IP : 3.95.254.165

26 апреля 2023 г., 05:49:05

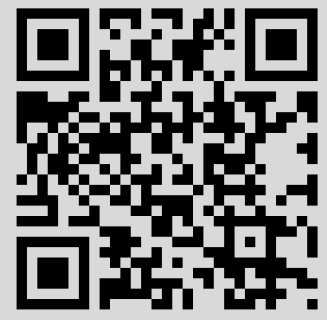




\title{
О МАКСИМАЛЬНЫХ ПАРОСОЧЕТАНИЯХ В $n$-МЕРНОМ КУБЕ
}

\section{В. Е. Тараканов}

\begin{abstract}
Решается задача эффективного нахождения максимальных паросочетаний в $n$-мерном кубе, имеющая применение в теории кодирования. Дается способ нахождения такого паросочетания при нечетном $n$ (теорема 2). Этот способ основан на построении в явном виде (теорема 1) таких отображений множества всех вершин, которые индуцируют наибольшие паросочетания во всяком двудольном подграфе $n$-мерного куба при любом $n$.

Библиография: 3 названия.
\end{abstract}

В работе решается одна из известных задач экстремальной комбинаторики. Рассматривается множество

$$
V_{n}=\left\{\left(\varepsilon_{0}, \varepsilon_{1}, \ldots, \varepsilon_{n-1}\right) \mid \varepsilon_{i} \in\{0,1\}\right\}
$$

всех $n$-мерных $(0,1)$-векторов, назьваемое $n$-мерным кубом. На $V_{n}$ как на множестве вершин задается графовая структура: векторы $x$ и $y$ из $V_{n}$ смежны тогда и только тогда, когда все, кроме одной, их координаты совпадают (другими словами, когда $d(x, y)=1$, где $d$ - расстояние Хэмминга). Таким образом, множество

$$
E=\left\{\{x, y\} \mid x, y \in V_{n}, d(x, y)=1\right\}
$$

представляет собой множество всех ребер $n$-мерного куба; условием (2) (вместе с (1)) полностью определена графовая структура $V_{n}$.

На $V_{n}$ естественным способом можно задать групповую операцию и умножение на 0 и 1 , которые превращают его в векторное пространство над полем $F=G F(2)$ из двух элементов. Соответствие

$$
x=\left(\varepsilon_{0}, \varepsilon_{1}, \ldots, \varepsilon_{n-1}\right) \in V_{n} \Longleftrightarrow x(z)=\sum_{i=0}^{n-1} \varepsilon_{i} z^{i}
$$

есть взаимно однозначное соответствие между $V_{n}$ и множеством $F^{(n-1)}[z]$ всех многочленов степени не выше $n-1$ от неизвестной $z$, сохраняющее сложение элементов. Фиксируя это соответствие, мы постоянно будем представлять $x \in V_{n}$ соответствующим многочленом из (3).

Работа выполнена при частичной поддержке гранта ведущих научных школ Российского фонда фундаментальных исследований, грант № 00-15-96135. 
Далее мы используем графовую терминологию из [1]. Паросочетанием в графе $G$ назовем любое независимое множество его ребер. Паросочетание $M$ максимально, если его нельзя дополнить никаким ребром. Наибольиим назьвается максимальное паросочетание, содержашее наибольшее возможное число ребер. Число $\beta_{1}(G)$ ребер в наибольшем паросочетании графа $G$ называется реберным числом независимости и является одной из основных теоретико-графовых констант. Экстремальная задача о паросочетаниях для различных классов графов - это

1) определение или оценка числа $\beta_{1}(G)$ для граффов $G$ данного класса и

2) построение алгоритмов для нахождения максимальных паросочетаний.

Как 1), так и, в особенности, 2) имеют эффективное решение лишь в отдельных частных случаях. Задача о максимальных паросочетаниях в $n$-мерном кубе представляет интерес в связи с построением корректирующих кодов (см. [2]). Мыпредставим здесь эффективньй алгоритм нахождения максимального паросочетания в графе $V_{n}$ при любом нечетном $n \geqslant 3$. Весом $w(x)$ вектора $x \in V_{n}$ назьваем число его ненулевых координат. Через $V_{n}^{(k)}$ обозначим совокупность всех векторов веса $k$. Ясно, что $V_{n}=\bigcup_{k=0}^{n} V_{n}^{(k)}$. Очевидно, что в $V_{n}$ могут быть смежны лишь векторы $x, y$ такие, что $x \in V_{n}^{(k)}, y \in$ $V_{n}^{(k+1)}, k=0,1, \ldots, n-1$. Через [ $\left.\alpha\right]$ обозначаем целую часть вешественного числа $\alpha$.

ПРЕДЛОЖЕНИЕ 1. Пусть $k$ - иелое число в отрезке $[0, n-1], G_{k}$ - подграф графа $V_{n}$ с множеством вершин $V_{n}^{(k)} \cup V_{n}^{(k+1)}$. Справедливо

$$
\beta_{1}\left(G_{k}\right)= \begin{cases}\left(\begin{array}{l}
n \\
k
\end{array}\right), & k<\left[\frac{n}{2}\right], \\
\left(\begin{array}{c}
n \\
k+1
\end{array}\right), & k>\left[\frac{n}{2}\right]\end{cases}
$$

где $\beta_{1}$ - число реберного покрытия графа.

ДокАЗАТЕЛьСТво. Действительно, $G_{k}$ - двудольный граф без изолированных вершин. Для такого графа число $\alpha_{0}$ вершинного покрытия равно величине его меньшей доли. Но по теореме Кенига [3] для всякого двудольного графа $G$ числа $\alpha_{0}(G)$ и $\beta_{1}(G)$ совпадают, и мы получаем наше утверждение.

Перейдем теперь к построению эффективного алгоритма нахождения наибольшего паросочетания в графах $G_{k}$ при $k<[n / 2]$. Воспользуемся представлением (3) для вершин $V_{n}$. Расположим многочлены из $F^{(n-1)}[z]$, имеющие одинаковое число ненулевых коэффищиентов (а, следовательно, и векторы с одинаковым весом), в лексикографическом порядке. А именно, для данных двух таких элементов

$$
x_{1}=\sum_{i=0}^{n-1} \varepsilon_{i}\left(x_{1}\right) z^{i} \quad \text { и } \quad x_{2}=\sum_{i=0}^{n-1} \varepsilon_{i}\left(x_{2}\right) z^{i}
$$

считаем $x_{1}>x_{2}$, если либо $\varepsilon_{0}\left(x_{1}\right)>\varepsilon_{0}\left(x_{2}\right)$, либо для некоторого $l \geqslant 0 \varepsilon_{k}\left(x_{1}\right)=\varepsilon_{k}\left(x_{2}\right)$, $k=0,1, \ldots, l, \varepsilon_{l+1}\left(x_{1}\right)>\varepsilon_{l+1}\left(x_{2}\right)$. 
Алгоритм. Строим однозначное соответствие $\varphi: V_{n}^{(k)} \rightarrow V_{n}^{(k+1)}$, беря последовательно элементы из $V_{n}^{(k)}$ во введенном нами лексикографическом порядке (по убьванию) и действуя по правилу: для $x=\sum_{i=0}^{n-1} \varepsilon_{i}(x) z^{i}$ пусть

$$
\varphi(x)=z^{j}+\sum_{i=0}^{n-1} \varepsilon_{i}(x) z^{i}=z^{j}+x
$$

где $j$ - наименьшее среди чисел $k$ со свойствами:

a) $\varepsilon_{k}(x)=0$

б) $z^{k}+\sum_{i=0}^{n-1} \varepsilon_{i}(x) z^{i} \neq \varphi\left(x^{\prime}\right)$ для $x^{\prime}>x$.

Мы установим в последующем, что:

1) действуя по алгоритму, мы действительно придем к наибольшему паросочетанию в $G_{k}$;

2 ) значение $\varphi(x)$ может быть найдено непосредственно по $x$, без всякой информации о значениях $\varphi\left(x^{\prime}\right)$ для $x^{\prime}>x$.

Свойство 2) обеспечивает эффективность сформулированного выше алгоритма.

Будем рассматривать конечные последовательности натуральных чисел. Множество всех таких последовательностей обозначаем через $N$.

Последовательность

$$
B=\left(b_{\alpha}, b_{\alpha+1}, \ldots, b_{\beta}\right)
$$

из $N$ назьвается сбалансированной, если

$$
\sum_{i=\alpha_{1}}^{\beta}\left(b_{i}-2\right) \leqslant 0
$$

для любого $\alpha_{1}$ такого, что $\alpha \leqslant \alpha_{1} \leqslant \beta$. Обозначим через $\Sigma(B)$ для $B \in N$ из (5) сумму (6) при $\alpha_{1}=\alpha$.

Примеры сбалансированных последовательностей $B \in N$ :

a) $(1,3,1,3,1,3,1)$;

б) $(2,4,1,2,1)$;

в) $(6,1,2,2,1,1,5,1,2,1,2,1,1,1,2)$.

Последовательность $B \in N$ из (5) назьвается строго сбалансированной, если она сбалансирована и $\Sigma(B)=0$. Таким образом, строго сбалансирована последовательность

$$
(2,2, \ldots, 2)
$$

а в приведенных вьше примерах - последовательность б); для а) и в) имеем $\Sigma(B)<0$ и они не являются строго сбалансированными. Отметим некоторые простые свойства сбалансированных последовательностей, нужные для дальнейшего.

Свойство 1. Если $B=\left(b_{\alpha}, b_{\alpha+1}, \ldots, b_{\beta}\right)$ из $N$ сбалансирована, то $b_{\beta} \leqslant 2$. 
Свойство 2. Пусть $B=\left(b_{\alpha}, b_{\alpha+1}, \ldots, b_{\beta}\right), B^{\prime}=\left(b_{\beta+1}, b_{\beta+2}, \ldots, b_{\beta^{\prime}}\right) \in N$ и $B$ сбалансирована. Тогда последовательность $B B^{\prime}=\left(b_{\alpha}, b_{\alpha+1}, \ldots, b_{\beta}, b_{\beta+1}, b_{\beta+2}, \ldots, b_{\beta^{\prime}}\right)$ сбалансирована в том и только том случае, когда сбалансирована $B^{\prime}$.

Свойство 3 . Если последовательность $B=\left(b_{\alpha}, b_{\alpha+1}, \ldots, b_{\beta}\right)$ из $N$ сбалансирована, но не строго сбалансирована, то либо

a) $b_{\beta}=2$ и найдется минимальньй индекс $\alpha_{1}$ такой, что $b_{\alpha_{1}}=1$ и $\left(b_{\alpha}, b_{\alpha+1}, \ldots, b_{\alpha_{1}}\right)$ сбалансирована, а $\left(b_{\alpha_{1}+1}, b_{\alpha_{1}+2}, \ldots, b_{\beta}\right)$ строго сбалансирована, либо

б) $b_{\beta}=1$ и $\left(b_{\alpha}, b_{\alpha+1}, \ldots, b_{\beta-1}\right)$ сбалансирована (или пуста).

Действительно, обозначим

$$
\Sigma_{\gamma}=\sum_{i=\gamma}^{\beta}\left(b_{j}-2\right)
$$

для целого $\gamma$ из отрезка $[\alpha, \beta]$. Имеем $\Sigma_{\alpha}<0$. Предположим, что найдется индекс $\alpha_{1}$ такой, что $\Sigma_{\alpha^{\prime}}<0$ для всех $\alpha^{\prime} \leqslant \alpha_{1}$ и $\Sigma_{\alpha_{1}+1}=0$. Так как

$$
\sum_{i=\alpha^{\prime}}^{\beta}\left(b_{i}-2\right)=\sum_{i=\alpha^{\prime}}^{\beta}\left(b_{i}-2\right)-\Sigma_{\alpha_{1}+1} \leqslant 0
$$

для всех $\alpha^{\prime}, \alpha \leqslant \alpha^{\prime} \leqslant \alpha_{1}$, то последовательность $\left(b_{\alpha}, b_{\alpha+1}, \ldots, b_{\alpha_{1}}\right)$ сбалансирована. Далее, $\left(b_{\alpha_{1}+1}, \ldots, b_{\beta}\right)$ сбалансирована и $\Sigma_{\alpha_{1}+1}=0$, поэтому она строго сбалансирована. Наконец, из $\Sigma_{\alpha_{1}}<0, \Sigma_{\alpha_{1}+1}=0$ следует, что $b_{\alpha_{1}}-2=-1$, т.е. $b_{\alpha_{1}}=1$.

Предположим теперь, что $\Sigma_{\alpha_{1}}<0$ для любого $\alpha_{1}, \alpha \leqslant \alpha_{1} \leqslant \beta$. Тогда $b_{\beta}=1$. Если $\alpha<\beta$ (т.е. множество $\left(b_{\alpha}, b_{\alpha+1}, \ldots, b_{\beta-1}\right)$ непусто), то для любого $\alpha_{1}$ в отрезке $[\alpha, \beta-1]$ имеем

$$
\sum_{i=\alpha_{1}}^{\beta-1}\left(b_{i}-2\right)=\Sigma_{\alpha_{1}}-\left(b_{\beta}-2\right)=\Sigma_{\alpha_{1}}+1 \leqslant 0 .
$$

Таким образом, последовательность $\left(b_{\alpha}, \ldots, b_{\beta-1}\right)$ сбалансирована, и наше утверждение доказано.

Свойство 4 . Если $B=\left(b_{\alpha}, b_{\alpha+1}, \ldots, b_{\beta}\right) \in N$ сбалансирована, но не строго сбалансирована и $b_{\beta}=1$, то при $\beta>\alpha$ последовательность $\left(b_{\alpha}, \ldots, b_{\beta-1}\right)$ сбалансирована.

Введем дополнительно несколько определений. Через $Z_{n}(m,<), m \leqslant n$, обозначим совокупность всех множеств $\left(c_{0}, c_{1}, \ldots, c_{m-1}\right), c_{i} \in Z_{n}=\{0,1, \ldots, n-1\}, i=0,1, \ldots$ $\ldots, m-1$, упорядоченных по возрастанию: $c_{0}<c_{1}<\cdots<c_{m-1}$. Для $C=\left(c_{0}, c_{1}, \ldots\right.$ $\left.\ldots, c_{m-1}\right) \in Z_{n}(m,<)$ обозначим

$$
C^{\prime}=\left(c_{1}^{\prime}, c_{2}^{\prime}, \ldots, c_{m-1}^{\prime}\right), \quad \text { где } c_{k}^{\prime}=c_{k}-c_{k-1}, k=1,2, \ldots, m-1 .
$$

Множество $C=\left(c_{0}, c_{1}, \ldots, c_{m-1}\right) \in Z_{n}(m,<)$ назовем специальным, если $m>1$ и для него последовательность $C^{\prime}$ из (8) сбалансирована или если $m=1$. Например, при $n=11, m=5$ множества $(0,3,6,7,8),(2,5,6,9,10)$ специальны, а множества $(0,1,4,7,8)$ или $(3,4,5,6,9)$ специальньми не являются. 
Специальной частью для $C=\left(c_{0}, c_{1}, \ldots, c_{m-1}\right) \in Z_{n}(m,<)$ назовем его специальное подмножество $C^{*}=\left(c_{0}, c_{1}, \ldots, c_{m^{*}}\right)$ такое, что $\left(c_{0}, c_{1}, \ldots, c_{s}\right)$ при $s>m^{*}$ не специально. Число $m^{*}=m^{*}(C)$ назовем индексом специальности для $C \in Z_{n}(m,<)$. Таким образом, для специального множества $C$ (т.е. когда $m^{*}(C)=m-1$ ) и только для него его специальная часть совпадает с ним самим. Нахождение индекса специальности $m^{*}(C)$ для $C=\left(c_{0}, c_{1}, \ldots, c_{m-1}\right)$ и соответствуюшего числа $c_{m *}$ сводится, очевидно, $\mathrm{K}$ несложным вычислениям по формуле (6) с элементами последовательности (8), построенной для $C$. Действительно, образуем подпоследовательности

$$
\left(c_{1}^{\prime}, c_{2}^{\prime}, \ldots, c_{s}^{\prime}\right), \quad s=1,2, \ldots
$$

Множество $\left(c_{0}\right)$ специально по определению. Далее, для последовательностей из (9) c $s=1,2, \ldots$ проверяем выполнение условий (6). Максимальное $s$, при котором (6) выполняются, и будет, очевидно, индексом специальности $m^{*}(C)$ - для него множество $\left(c_{0}, c_{1}, \ldots, c_{s}\right)$ специально, а $\left(c_{0}, c_{1}, \ldots, c_{s^{\prime}}\right)$ при $s^{\prime}>s$ специальным быть не может. Итак, мы пришли к следующему результату.

ПРЕДЛОЖЕНИЕ 2. Пусть $n$ - натуральное число, $m \in Z_{n}, C=\left(c_{0}, c_{1}, \ldots, c_{m-1}\right) \in$ $Z_{n}(m,<)$. Обозначим $c_{i}^{\prime \prime}=c_{i}-c_{i-1}-2, i=1, \ldots, m-1$. Тогда

$$
m^{*}= \begin{cases}\max \left\{s \mid \sum_{i=\alpha}^{s} c_{i}^{\prime \prime} \leqslant 0, \alpha=0,1, \ldots, s\right\}, & \text { если такое s существует, } \\ 0 & \text { в противном случае }\end{cases}
$$

где $m^{*}=m^{*}(C)-$ индекс специальности для $C$.

Сформулируем одно вспомогательное утверждение.

Лемма 1. Пусть $n$ - натуральное число, $m$ - иелое в отрезке $[2,[n / 2]]$. Множество $C=\left(c_{0}, c_{1}, \ldots, c_{m-1}\right) \in Z_{n}(m,<)$ мохет быть специальным лииь при $c_{m-1}-c_{0} \leqslant 2(m-1)$.

ДокАЗАТЕЛьСтво. Действительно, по определению специального множества

$$
c_{m-1}-c_{0}=\sum_{k=1}^{m-1} c^{\prime} k \leqslant 2(m-1) .
$$

Пусть

$$
x=\sum_{i=0}^{n-1} \varepsilon_{i}(x) z^{i}
$$

- элемент $V_{n}$ в представлении (3). Если $w(x)=m$, то определим

$$
S(x)=C=\left\{c_{0}, c_{1}, \ldots, c_{m-1}\right\}
$$

где $C \in Z_{n}(m,<)$ - совокупность всех тех индексов $i$, для которых $\varepsilon_{i}(x) \neq 0$. Тогда имеем представление для $x \in V_{n}^{(m)}$ :

$$
x=\sum_{c_{i} \in S(x)} z^{c_{i}}
$$


где $S(x)$ определено в (11).

Перейдем теперь к детальному рассмотрению сформулированного вьше алгоритма построения наибольшего паросочетания в подграфе $G_{k} n$-мерного куба $V_{n}(k \leqslant[n / 2])$. Введем еще одно обозначение. Для $C=\left(c_{0}, c_{1}, \ldots, c_{m-1}\right) \in Z_{n}(m,<)$ обозначим

$$
\widehat{C}^{(i)}=\left(c_{0}, c_{1}, \ldots, c_{i-1}, c_{i+1}, \ldots, c_{m-1}\right)
$$

упорядоченное множество $C$, из которого удален элемент $c_{i}(0 \leqslant i \leqslant m-1)$. Пусть также

$$
r= \begin{cases}\frac{n-1}{2}, & n \text { нечетно, } \\ \frac{n}{2}, & n \text { четно. }\end{cases}
$$

ТЕОрема 1. Пусть $n$ - натуральное число, $m$ - иелое в отрезке $[1, r]$. Предположим, что задан вектор х веса тиз $V_{n}$ и $x=\sum_{c_{i} \in S(x)} z^{c_{i}}$ - его представление (12) $\left(S(x)=C=\left(c_{0}, c_{1}, \ldots, c_{m-1}\right)\right.$ определено в $\left.(11)\right)$. Пусть $m^{*}=m^{*}(S(x))-$ индекс спеииальности множества $S(x)$. Тогда определим

$$
\psi(x)= \begin{cases}z^{c_{m^{*}+1}}+x, & \text { если } m^{*}<m-1 \text { или } m^{*}=m-1 \text { u } c_{m-1}<n-1 \\ 1+x, & \text { если } m^{*}=m-1 \text { u } c_{m-1}=n-1\end{cases}
$$

Если $\varphi$ - соответствие, определяемое правилом (4), то значение $\varphi(x)$ определено $u \varphi(x)=\psi(x)$.

ДокАЗАТЕЛЬСТво. Будем доказывать теорему (в целях большей прозрачности доказательства) лишь в “критическом" случае - для нечетного $n$ и $m=r=(n-1) / 2$. В остальных случаях доказательство проводится совершенно аналогично.

Итак, пусть $m=r$. Заметим прежде всего, что если множество $C$ не специально (т.е. если $\left.m^{*}<r-1\right)$, то по свойству $1 c_{m^{*}+1}-c_{m^{*}} \geqslant 3$ и, следовательно, $c_{m^{*}}+1 \notin S(x)$. Ясно, что несколько начальных шагов при установлении соответствия $\varphi$ всегда можно сделать и что при этом получается $\varphi(x)=\psi(x)$ для нескольких “самых больших" элементов из $V_{n}^{(r)}$. Далее применяем принцип математической индукции, предполагая, что при рассмотрении элемента $x$ мы уже знаем, что $\varphi(y)=\psi(y)$ для всех $y>x$. Мы получим доказательство теоремы в виде следствия нескольких вспомогательных утверждений.

ЛЕмма 2. Пусть $c$ - любое иелое число в интервале $\left(c_{0}, c_{m^{*}}\right)$ такое, что с $\notin$ $S(x)$. Тогда найдется әлемент $y \in V_{n}^{(r)}$ такой, что $\varphi(y)=\psi(y)=z^{c}+x$.

ДоказАтЕльство. Определим $k$ как наименьшее число такое, что $c_{k-1}<c<c_{k}$. Рассмотрим множества $D=\left(d_{0}, d_{1}, \ldots, d_{r}\right)$ и $D^{*}=\left(d_{0}, d_{1}, \ldots, d_{m^{*}+1}\right)$, где

$$
d_{i}= \begin{cases}c_{i}, & 0 \leqslant i \leqslant k-1 \\ c, & i=k, \\ c_{i-1}, & k+1 \leqslant i \leqslant m-1\end{cases}
$$


а также последовательность $\left(D^{*}\right)^{\prime}=\left(d_{1}^{\prime}, d_{2}^{\prime}, \ldots, d_{m^{*}+1}^{\prime}\right), d_{i}^{\prime}=d_{i}-d_{i-1}, i=1,2, \ldots$ $\ldots, m^{*}+1$, определенную для множества $D^{*}$. Ясно, что $D^{*}$ специально и $\left(D^{*}\right)^{\prime}$ сбалансирована. Имеем

$$
d_{k+1}^{\prime}=c_{k}-c \leqslant c_{k}^{\prime}-1
$$

а также

$$
d_{k}^{\prime}-2+d_{k+1}^{\prime}-2=c_{k}^{\prime}-4 .
$$

Из (17) следует, что $\left(D^{*}\right)^{\prime}$ не является строго сбалансированной.

Рассмотрим сбалансированную подпоследовательность $\left(d_{k+1}^{\prime}, \ldots, d_{m^{*}+1}^{\prime}\right)$ последовательности $\left(D^{*}\right)^{\prime}$. Ввиду (16) она не является строго сбалансированной. По свойству 3 существуют всего две возможности:

А) найдется минимальньй индекс $j \geqslant k+1$ такой, что

a) $d_{j}^{\prime}=1$

б) $\left(d_{k+1}^{\prime}, \ldots, d_{j}^{\prime}\right)$ сбалансирована,

в) $\left(d_{j+1}^{\prime}, \ldots, d_{m^{*}+1}^{\prime}\right)$ строго сбалансирована;

Б) $d_{m^{*}+1}=1$ и $\left(d_{k+1}^{\prime}, \ldots, d_{m^{*}}^{\prime}\right)$ сбалансирована.

В случае Б) полагаем $j=m^{*}+1$.

Разберем случаи А) и Б) по отдельности и покажем, что в каждом случае множество $\left(d_{0}, d_{1}, \ldots, d_{j-1}\right)$ специально.

Случай А). Ввиду строгой сбалансированности последовательности $\left(d_{j+1}^{\prime}, \ldots\right.$ $\left.\ldots, d_{m^{*}+1}^{\prime}\right)=\left(c_{j}^{\prime}, \ldots, c_{m^{*}}^{\prime}\right)$ последовательности $\left(c_{1}^{\prime}, \ldots, c_{j-1}^{\prime}\right),\left(c_{k}^{\prime}, \ldots, c_{j-1}^{\prime}\right)$ и соответственно $\left(d_{1}^{\prime}, \ldots, d_{j}^{\prime}\right),\left(d_{k+1}^{\prime}, \ldots, d_{j}^{\prime}\right)$ сбалансированы.

Разберем по отдельности два случая.

$\left.\mathrm{A}_{1}\right) c_{k}^{\prime} \geqslant 3$. Так как $c_{j-1}^{\prime}=d_{j}^{\prime}=1, j \neq k+1$, и имеем $j \geqslant k+2$. Для сбалансированной подпоследовательности

$$
\left(c_{k+1}^{\prime}, c_{k+2}^{\prime}, \ldots, c_{j-1}^{\prime}\right)
$$

последовательности $\left(c_{k}^{\prime}, \ldots, c_{j-1}^{\prime}\right)$ справедливо

$$
\sum_{i=k+1}^{j-1}\left(c_{i}^{\prime}-2\right)=\sum_{i=k}^{j-1}\left(c_{i}-2\right)-\left(c_{k}^{\prime}-2\right) \leqslant-1 .
$$

Следовательно, $(18)$ не строго сбалансирована. Поэтому последовательность $\left(c_{k+1}^{\prime}, \ldots\right.$ $\ldots, c_{j-2}^{\prime}$ ), если она непуста (что имеет место при $j \geqslant k+3$ ), по свойству 4 является сбалансированной, т.е.

$$
\sum_{i=\alpha}^{j-2}\left(c_{i}^{\prime}-2\right) \leqslant 0, \quad k+1 \leqslant \alpha \leqslant j-2 .
$$

Далее, ввиду сбалансированности $\left(c_{1}^{\prime}, \ldots, c_{j-1}^{\prime}\right)$ имеем при $1 \leqslant \alpha \leqslant k$

$$
\sum_{i=\alpha}^{j-2}\left(c_{i}^{\prime}-2\right)=\sum_{i=\alpha}^{j-1}\left(c_{i}^{\prime}-2\right)-\left(c_{j-1}^{\prime}-2\right) \leqslant 1 .
$$


Таким образом,

$$
\sum_{i=\alpha}^{j-2}\left(c_{i}^{\prime}-2\right) \leqslant \begin{cases}1, & 1 \leqslant \alpha \leqslant k \\ 0, & k+1 \leqslant \alpha \leqslant j-2, j \geqslant k+3 .\end{cases}
$$

Отсюда, учитывая (16) и (17), получаем

$$
\begin{gathered}
\sum_{i=\alpha}^{j-1}\left(d_{i}^{\prime}-2\right)=-2+\sum_{i=\alpha}^{j-2}\left(c_{i}^{\prime}-2\right) \leqslant-1, \quad 1 \leqslant \alpha \leqslant k, \\
\sum_{i=k+1}^{j-1}\left(d_{i}^{\prime}-2\right) \leqslant-1+\sum_{i=k}^{j-2}\left(c_{i}^{\prime}-2\right) \leqslant 0, \\
\sum_{i=\alpha}^{j-1}\left(d_{i}^{\prime}-2\right)=\sum_{i=\alpha-1}^{j-2}\left(c_{i}^{\prime}-2\right) \leqslant 0, \quad k+2 \leqslant \alpha \leqslant j-1 .
\end{gathered}
$$

Тем самьм, $\sum_{i=\alpha}^{j-1}\left(d_{i}^{\prime}-2\right) \leqslant 0$ для любого $\alpha, 1 \leqslant \alpha \leqslant j-1$. Таким образом, последовательность $\left(d_{1}^{\prime}, \ldots, d_{j-1}^{\prime}\right)$ сбалансирована и, следовательно, $\left(d_{0}, d_{1}, \ldots, d_{j-1}\right)$ специальHO.

$\left.\mathrm{A}_{2}\right) c_{k}^{\prime}=2$. Если $j \geqslant k+2$, то (18) не может быть строго сбалансированной - в противном случае по свойству 2 была бы строго сбалансированной последовательность $\left(c_{k+1}^{\prime}, \ldots, c_{m^{*}}^{\prime}\right)=\left(d_{k+2}^{\prime}, \ldots, d_{m^{*}+1}^{\prime}\right)$ и получилось бы противоречие с минимальностью $j$. Ввиду этого при $j \geqslant k+2$ мы можем вьвести свойство специальности множества $\left(d_{0}, d_{1}, \ldots, d_{j-1}\right)$, рассуждая в точности так же, как в $\left.A_{1}\right)$.

Пусть $j=k+1$. Последовательность $\left(d_{k+2}^{\prime}, \ldots, d_{m^{*}+1}^{\prime}\right)$ строго сбалансирована. Поэтому $\left(d_{1}^{\prime}, \ldots, d_{k+1}^{\prime}\right)$ сбалансирована, но не строго сбалансирована (иначе строго сбалансированной была бы $\left.\left(D^{*}\right)^{\prime}\right)$, и при этом $d_{k+1}^{\prime}=d_{j}^{\prime}=1$. По свойству 4 тогда сбалансирована последовательность $\left(d_{1}^{\prime}, \ldots, d_{k}^{\prime}\right)$, и, таким образом, множество $\left(d_{0}, d_{1}, \ldots\right.$ $\left.\ldots, d_{j-1}\right)$ специально.

Случай Б). $d_{m^{*}+1}^{\prime}=1,\left(d_{k+1}^{\prime}, \ldots, d_{m^{*}}^{\prime}\right)$ сбалансирована. По условию (см. вьше) $j=m^{*}+1$. Так как $\left(D^{*}\right)^{\prime}$ сбалансирована, но не строго сбалансирована и $d_{m^{*}+1}^{\prime}=1$, по свойству $4\left(d_{1}^{\prime}, \ldots, d_{m^{*}}^{\prime}\right)$ сбалансирована. Следовательно, $\left(d_{0}, d_{1}, \ldots, d_{j-1}\right)$ специально.

Итак, мы показали, что как в случае А), так и в случае Б) $\left(d_{0}, d_{1}, \ldots, d_{j-1}\right)$ специально. Рассмотрим теперь множество $\widehat{D}^{(j)}$ (см. (13)) и соответствующие последовательности

$$
\begin{gathered}
\left(d_{1}^{\prime}, d_{2}^{\prime}, \ldots, d_{j-1}^{\prime}, d_{j+1}-d_{j-1}\right), \\
\left(d_{1}^{\prime}, d_{2}^{\prime}, \ldots, d_{j-1}^{\prime}, d_{j+1}-d_{j-1}, d_{j+2}^{\prime}, \ldots, d_{p}^{\prime}\right), \quad j+2 \leqslant p \leqslant r .
\end{gathered}
$$

Мы покажем, что специальная часть $\widehat{D}^{(j)}$ - это $\left(d_{0}, d_{1}, \ldots, d_{j-1}\right)$.

Если $m^{*}=r-1$, т.е. $m^{*}+1=r$ и $d_{m^{*}+1}^{\prime}=1$, то свойство специальности множества $\left(d_{0}, d_{1}, \ldots, d_{r-1}\right)$ уже показано выше (см. случай Б)).

Далее пусть $m^{*}<r-1$. Заметим, что при любом $p, m^{*}+1<p \leqslant r$, последовательность $\left(d_{m^{*}+2}^{\prime}, \ldots, d_{p}^{\prime}\right)=\left(c_{m^{*}+1}^{\prime}, \ldots, c_{p-1}^{\prime}\right)$ не сбалансирована - иначе (по свойству 2$)$ 
была бы сбалансирована $\left(c_{1}^{\prime}, \ldots, c_{m^{*}}^{\prime}, c_{m^{*}+1}^{\prime}, \ldots, c_{p-1}^{\prime}\right)$, что противоречит $m^{*}(C)=m^{*}$. Нетрудно также заметить, что последовательность $\left(d_{j+1}-d_{j-1}, d_{j+2}^{\prime}, \ldots, d_{m *+1}^{\prime}\right)$ не сбалансирована: при $j<m^{*}+1$ это следует из строгой сбалансированности $\left(d_{j+1}^{\prime}\right.$, $\left.d_{j+2}^{\prime}, \ldots, d_{m^{*}+1}^{\prime}\right)$; при $j=m^{*}+1$ для единственного члена имеем $d_{j+1}-d_{j-1}=d_{j}-$ $d_{j-1}+d_{j+1}-d_{j} \geqslant 4$. Поэтому каждая из последовательностей (19) имеет несбалансированный "хвост" (начиная с члена $d_{j+1}-d_{j-1}$ ) и, следовательно, сама не сбалансирована. Значит, соответствующие множества $\left(d_{0}, d_{1}, \ldots, d_{j+1}-d_{j-1}\right),\left(d_{0}, d_{1}, \ldots, d_{j-1}\right.$, $\left.d_{j+1}, \ldots, d_{p}\right), j+2 \leqslant p \leqslant r$, не могут быть специальными, и во всех случаях специальная часть множества $\widehat{D}^{(j)}$ есть $\left(d_{0}, d_{1}, \ldots, d_{j-1}\right)$, т.е.

$$
m^{*}\left(\widehat{D}^{(j)}\right)=j-1
$$

Возьмем теперь элемент $y$ с $S(y)=\widehat{D}^{(j)}$. Ясно, что $y \in V_{n}^{(r)}$ и что $y>x$. По предположению индукции и ввиду $(20) \varphi(y)=\psi(y)=z^{d_{j-1}+1}+y=z^{d_{j}}+1$, так как $d_{j}-d_{j-1}=1$. Так как $j \geqslant k+1$, имеем $d_{j}=c_{j-1}$. Тем самым, $\varphi(y)=\psi(y)=z^{c_{j-1}}+y=$ $z^{c}+x$. Лемма доказана.

ЛЕмма 3. Пусть $x=z^{c_{0}}+z^{c_{1}}+\cdots+z^{c_{r-1}}$ - әлемент из $V_{n}^{(r)}$ в представлении (12) с $S(x)=C=\left(c_{0}, c_{1}, \ldots, c_{r-1}\right), m^{*}(S(x))=m^{*}-$ индекс специальносmu $S(x)$. Если $y>x$, mо $\varphi(y) \neq x+z^{c_{m^{*}+1}}$.

ДокАЗАТЕЛЬСТво. Предположим, от противного, что $\varphi(y)=\psi(y)=z^{c_{m}+1}+x$ для некоторого $y>x$. Этот $y$ может отличаться от $x$ лишь двумя членами: в $y$ входит $z^{c} m^{*+1}$ и не входит $z^{c}$ для некоторого $c \in S(x)$. При этом $c \neq c_{0}, c_{1}, \ldots, c_{m^{*}}-$ иначе было бы $y<x$. Таким образом,

$$
S(y)=C_{1}=\left(c_{0}, c_{1}, \ldots, c_{m^{*}}, c_{m^{*}}+1, \ldots, c_{r-1}\right)-\left\{c_{k}\right\}
$$

для некоторого $k, m^{*}<k \leqslant r-1$. Кроме того, согласно формуле (14):

a) $c_{k}^{\prime}=1$;

б) множество $C_{1}^{*}=\left(c_{0}, c_{1}, \ldots, c_{m^{*}}, c_{m^{*}}+1, c_{m^{*}+1}, \ldots, c_{k-1}\right)-$ специальная часть $C_{1}$. Однако, если $C_{1}^{*}$ специально, то последовательность $\left(c_{1}^{\prime}, \ldots, c_{m^{*}}^{\prime}, 1, c_{m^{*}+1}-1, \ldots, c_{k-1}^{\prime}\right)$ сбалансирована; следовательно, сбалансированной будет также последовательность $\left(c_{m^{*}+1}-1, c_{m^{*}+2}^{\prime}, \ldots, c_{k-1}^{\prime}\right)$. Но тогда, как непосредственно проверяется по (6), сбалансирована также последовательность $\left(c_{m^{*}+1}^{\prime}, c_{m^{*}+2}^{\prime}, \ldots, c_{k}^{\prime}\right)$. По свойству 2 отсюда следует сбалансированность последовательности $\left(c_{1}^{\prime}, c_{2}^{\prime}, \ldots, c_{k}^{\prime}\right)$, и множество $\left(c_{0}, c_{1}, \ldots\right.$ $\ldots, c_{k}$ ) оказьвается специальньм. Но ввиду $m^{*}<k$ это противоречит равенству $m^{*}(S(x))=m^{*}$. Полученное противоречие доказьвает утверждение леммы.

Из лемм 2 и 3 теперь без труда получается утверждение теоремы в случаях, когда множество $S(x)$ не специально или когда оно специально и $c_{r-1}<n-1$. В самом деле, согласно лемме $2 \varphi(x)$ неможет равняться $z^{c}+x$ при $c<c_{m^{*}}$, так как все такие значения "заняты" элементами $\varphi(y)$ при каком-либо $y>x$. Таким образом, согласно правилу (4) $\varphi(x)=z^{c} m^{*+1}+x=\psi(x)$, если это значение еще не “занято". Но по лемме 3 оно действительно "свободно".

Рассмотрим теперь оставшийся случай, когда $S(x)$ специально и $c_{r-1}=n-1$. 
Лемма 4. Пусть множество $C=\left(c_{0}, \ldots, c_{r-1}\right) c c_{r-1}=n-1$ специально $и$

$$
x=\sum_{c_{i} \in C} z^{c_{i}} \in V_{n}^{(r)} .
$$

Не существует әлемента $y \in V_{n}^{(r)}, y>x$, для которого $\varphi(y)=\psi(y)=1+x$.

ДокАЗАТЕЛЬСтво. Пусть, от противного, такой $y$ найдется. Тогда $S(y)=(C \cup\{0\})$ $-\{c\}$ для некоторого элемента $c \in C$; при этом $c-1 \in C$ и $\left(0, c_{0}, c_{1}, \ldots, c-1\right)-$ специальная часть $S(y)$.

Предположим сначала, что $c=c_{r-1}=n-1$. Тогда $S(y)=\left(0, c_{0}, c_{1}, \ldots, c_{r-2}\right)$ специально и $c_{r-2}=n-2$. Однако по лемме 1 в любом специальном множестве наибольший элемент отстоит от наименьшего не более, чем на $n-3$. Таким образом, $S(y)$ не может быть специальньм.

Пусть $S(y)$ не специально. Также по лемме $1 c_{0} \geqslant 2$. Таким образом, $S(y)=\left(0, c_{0}\right.$, $\left.c_{1}, \ldots, c_{t}, c_{t+2}, \ldots, c_{r-1}\right)$ для некоторого $t, 1 \leqslant t<r-2, c_{0} \geqslant 2, c_{r-1}=n-1$, $c_{t+1}-c_{t}=1$, и множество $\left(0, c_{0}, c_{1}, \ldots, c_{t}\right)$ - специальная часть $S(y)$; лишь в таком случае $\psi(y)=1+x$. Поэтому последовательность $C_{1}^{\prime}=\left(c_{0}, c_{1}^{\prime}, \ldots, c_{t}^{\prime}\right)$ сбалансирована. Кроме того, ввиду специальности $C$ сбалансирована также последовательность $C_{2}^{\prime}=\left(c_{t+2}^{\prime}, \ldots, c_{r-1}^{\prime}\right)$. Для $C_{1}^{\prime}$ и соответственно $C_{2}^{\prime}$ справедливы неравенства

$$
c_{0}-2+\sum_{i=1}^{t}\left(c_{i}^{\prime}-2\right) \leqslant 0, \quad \sum_{i=t+2}^{r-1}\left(c_{i}^{\prime}-2\right) \leqslant 0 .
$$

Складывая неравенства и учитывая, что $c_{t+1}^{\prime}=c_{t+1}-c_{t}=1$, получаем $c_{r-1}-1 \leqslant 2 r-2$ или $c_{r-1} \leqslant n-2$, что противоречит условию $c_{r-1}=n-1$. Лемма доказана.

Из леммы 4 вытекает, что при специальном множестве $S(x)$ вида $\left(c_{0}, c_{1}, \ldots, c_{r-2}\right.$, $n-1)$, действуя по правилу 4 , мы должны в качестве $\varphi(x)$ взять значение $1+x=\psi(x)$. Этим завершается доказательство теоремы 1.

СлеДСтвиЕ. Пусть $n$ нечетно, $r=(n-1) / 2$. Формулой (14) устанавливается взаимно однозначное соответствие $x \rightarrow \psi(x)$ между множествами $V_{n}^{(r)}$ $u V_{n}^{(r+1)}$ всех п-мерных двоичных векторов весов $r$ u $r+1$ соответственно; при этом $d(x, \psi(x))=1$ для любого $x \in V_{n}^{(r)}$, где $d$ - расстояние Хәмминга.

Если $y=\sum_{c_{i} \in D} z^{c_{i}}-$ представление $y \in V_{n}^{(r+1)}$ в виде (12), где $D=S(y)$, то

$$
x=\psi^{-1}(y)=\sum_{c_{i} \in C} z^{c_{i}},
$$

где $C=S(x)=\widehat{D}^{(k)}$ и $k$ - наибольший среди индексов $l$ со свойствами: $c_{l}-c_{l-1}=1$, $\left(c_{0}, c_{1}, \ldots, c_{l-1}\right)$ - специальное множество.

Утверждение непосредственно следует из формулы (14).

Формула (10) позволяет без труда вычислять индекс специальности для множества $S(x)=\left(c_{0}, c_{1}, \ldots, c_{m-1}\right)$, с помошью которого задается вектор $x \in V_{n}^{(m)}$ веса $m$, $1 \leqslant m \leqslant[n / 2]$. Тем самым, по формуле (14) эффективно вычисляется образ $\psi(x)=\varphi(x)$ при рассматриваемом взаимно однозначном соответствии $V_{n}^{(m)} \rightarrow V_{n}^{(m+1)}$.

Применим полученные результаты к задаче эффективного нахождения максимального паросочетания в $n$-мерном кубе. 
ТЕОРема 2. Пусть $n \geqslant 3$ - нечетное иелое число, $V_{n}-n$-мерный куб с множеством ребер $E$ (см. (1), (2)). В Е мохсно әффективно построить максимальное паросочетание $M_{n}$, состоящее из $m_{n}$ ребер, где

$$
m_{n}=\left\{\begin{array}{ll}
\left(\begin{array}{l}
n \\
r
\end{array}\right)+\sum_{i=0}^{(r-2) / 2}\left(\begin{array}{l}
n \\
2 i
\end{array}\right) & \text { npu } n \equiv 1(\bmod 4), \\
\left(\begin{array}{l}
n \\
r
\end{array}\right)+\sum_{i=1}^{(r-1) / 2}\left(\begin{array}{c}
n \\
2 i-1
\end{array}\right) & \text { npu } n \equiv 3(\bmod 4),
\end{array} \quad r=\frac{n-1}{2} .\right.
$$

ДокАЗАТЕЛЬСтво. В $n$-мерном кубе две вершины $x$ и $y$ могут быть соединены ребром лишь в том случае, когда $|w(x)-w(y)|=1$. Строим максимальное паросочетание, используя найденное в теореме 1 отображение $\psi$, которое при $1 \leqslant m \leqslant r$ каждому $x \in V_{n}^{(m)}$ ставит в соответствие по формуле (14) элемент $\psi(x) \in V_{n}^{(m+1)}$. Доопределим отображение $\psi$ на единственном элементе веса 0:

$$
\psi(0)=1
$$

Тем самым, $\psi$ можно рассматривать как отображение $V_{n}^{(0)} \cup V_{n}^{(1)} \cup \cdots \cup V_{n}^{(r)}$ в $V_{n}^{(1)} \cup$ $V_{n}^{(2)} \cup \cdots \cup V_{n}^{(r+1)}$. Определим теперь на $V_{n}$ отображение $x \rightarrow \bar{x}$ :

$$
x=\sum_{i=0}^{n-1} \varepsilon_{i} z^{i} \rightarrow \bar{x}=\sum_{i=0}^{n-1} \bar{\varepsilon}_{i} z^{i}
$$

где $\bar{\varepsilon}_{i}=1-\varepsilon_{i}, i=0,1, \ldots, n-1$. Ясно, что если $w(x)=m$, то $w(\bar{x})=n-m$, $m=0,1, \ldots, n$. Пользуясь $(22)$ и $(23)$, распространим функцию $\psi(x)$ на элементы $x$ веса $w(x)>n / 2$ :

$$
\psi(x)=\overline{\psi(\bar{x})}
$$

при (24) $V_{n}^{(m)}$ отображается в $V_{n}^{(m-1)}, m=n-r, n-r+1, \ldots, n$, и вновь $d(x, \psi(x))=1$.

Строим паросочетание $M_{n}$, беря при $n \equiv 1(\bmod 4)$ ребра $(x, \psi(x))$ для всех $x \in V_{n}^{(i)}$, $i=0,1, \ldots, r / 2$, и для всех $x \in V_{n}^{(2 i+1)}, i=r / 2+1, r / 2+2, \ldots, r$. При $n \equiv 3(\bmod 4)$ берем $(x, \psi(x))$ для всех $x \in V_{n}^{(2 i-1)}, i=1,2, \ldots,(r+1) / 2$, и для всех $x \in V_{n}^{(2 i)}$, $i=(r+3) / 2,(r+5) / 2, \ldots, r$. В полученных множествах $M_{n}$ любая пара ребер не имеет общих конщов, поэтому $M_{n}$ - действительно паросочетание. Оно является максимальным. В самом деле, пусть $(x, y)$ - любое ребро из $E-M_{n}, x \in V_{n}^{(m)}, y \in V_{n}^{(m+1)}$, $0 \leqslant m \leqslant n-1$. Очевидно, что один из концов ребра $(x, y)$ принадлежит одному из тех множеств $V_{n}^{(k)}$, которые были взяты нами при построении $M_{n}$. Скажем, если $n \equiv 1$ $(\bmod 4), x \in V_{n}^{(r+1)}, y \in V_{n}^{(r+2)}$, то в $M_{n}$ обязательно найдется ребро $(u, x)$, где $u=$ $\psi^{-1}(x) \in V_{n}^{(r)}$. Таким образом, паросочетание $M_{n}$ действительно максимально. При $n \equiv 1(\bmod 4)$ оно состоит из

$$
\left(\begin{array}{l}
n \\
r
\end{array}\right)+\sum_{i=0}^{r / 2-1}\left(\begin{array}{l}
n \\
2 i
\end{array}\right)+\sum_{i=r / 2+1}^{r}\left(\begin{array}{c}
n \\
2 i+1
\end{array}\right)=\left(\begin{array}{l}
n \\
r
\end{array}\right)+2 \sum_{i=0}^{r / 2-1}\left(\begin{array}{l}
n \\
2 i
\end{array}\right)
$$


ребер. При $n \equiv 3(\bmod 4) M_{n}$ состоит из

$$
\left(\begin{array}{l}
n \\
r
\end{array}\right)+\sum_{i=1}^{(r-1) / 2}\left(\begin{array}{c}
n \\
2 i-1
\end{array}\right)+\sum_{i=(r+3) / 2}^{n-1}\left(\begin{array}{l}
n \\
2 i
\end{array}\right)=\left(\begin{array}{l}
n \\
r
\end{array}\right)+2 \sum_{i=1}^{(r-1) / 2}\left(\begin{array}{c}
n \\
2 i-1
\end{array}\right)
$$

ребер.

Значение $\psi(x)$ вычисляется по $x$ с помощью формул (10) и (14) за время, полиномиально зависящее от $n$. Тем самым, $M_{n}$ находится эффективно. Теорема доказана.

СлЕДСТВИЕ. Для реберного числа независимости $\beta_{1}\left(V_{n}\right)$-мерного куба $V_{n} n p и$ данном нечетном $n \geqslant 3$ справедливо неравенство

$$
\beta_{1}\left(V_{n}\right) \geqslant m_{n}
$$

где $m_{n}-$ число из (21).

В заключение отметим, что аналогичный теореме 2 результат для четного $n$ требует, по-видимому, более детального рассмотрения множества образов элементов $V_{n}$ при отображении $\psi$ из теоремы 1.

\section{СПИСОК ЦИТИРОВАННОЙ ЛИТЕРАТУРЫ}

[1] Харари Ф. Теория графов. М.: Мир, 1973.

[2] Камерон П., ван Линт Дж. Теория графов, теория кодирования и блок-схемы. М.: Наука, 1980.

[3] König D. Graphen und Matrizen // Mat. Fiz. Lapok. 1931. V. 38. P. 116-119. 